\title{
THE NATURE OF THE GOULD BELT FROM A FRACTAL ANALYSIS OF ITS STELLAR POPULATION \\ Néstor Sánchez, ${ }^{1}$ Emilio J. Alfaro, ${ }^{1}$ Federico Elias, ${ }^{2}$ Antonio J. Delgado, ${ }^{1}$ and Jesús Cabrera-Caño ${ }^{3}$ Received 2007 April 23; accepted 2007 June 9
}

\begin{abstract}
The Gould Belt (GB) is a system of gas and young, bright stars distributed along a plane that is inclined with respect to the main plane of the Milky Way. Observational evidence suggests that the GB is our closest star formation complex, but its true nature and origin remain rather controversial. In this work we analyze the fractal structure of the stellar component of the GB. In order to do this, we tailor and apply an algorithm that estimates the fractal dimension in a precise and accurate way, avoiding both boundary and small-data set problems. We find that early OB stars (of spectral types earlier than B4) in the GB have a fractal dimension very similar to that of the gas clouds in our Galaxy. On the contrary, stars in the GB of later spectral types show a larger fractal dimension, similar to that found for OB stars of both age groups in the local Galactic disk (LGD). This result seems to indicate that while the younger OB stars in the GB preserve the memory of the spatial structure of the cloud where they were born, older stars are distributed following a similar morphology as that found for the LGD stars. The possible causes for these differences are discussed.
\end{abstract}

Subject headings: methods: numerical — solar neighborhood — stars: early-type

\section{INTRODUCTION}

The brightest stars near the Sun are mainly distributed along two great circles over the sky: the Milky Way plane and another strip of bright stars known as the Gould Belt (GB), which is inclined with respect to the Galactic plane (Gould 1879; Stothers \& Frogel 1974). Although the existence of the GB was formally reported as soon as in 1847 by Sir John F. W. Herschel (1847) from naked-eye observations of the southern sky, its nature and origin are still poorly understood. The GB is a complex system of gas and stars composed not only by single massive stars but also by OB associations and an interstellar medium (dust, neutral hydrogen, and molecular clouds) that shows some kinetic features which have been considered by several authors as connected to the stellar population (Lindblad et al. 1973). The GB was first interpreted as an apparently expanding ring (or torus) of young massive stars, but later, with the detection of young low-mass stars, its structure seemed to be better represented by an inhomogeneous disk (Guillout et al. 1998). Recent studies characterize it as a stellar disk having elliptical shape, with a semimajor axis of $\sim 600 \mathrm{pc}$ and semiminor axis of $\sim 400 \mathrm{pc}$, which is inclined around $18^{\circ}$ to the Galactic plane (Elias et al. 2006b). The stellar component exhibits a range of ages lesser than 100 million years (Torra et al. $2000)$. Given its size $(\sim 1 \mathrm{kpc})$, age range, and relationship with the gas in different phases, it has been suggested that the GB would be our closest star formation complex (Elmegreen et al. 2000), in the sense given to this concept by Efremov (1995), i.e., the largest region of a galaxy showing a coherent star formation process, where the term "coherent" should be interpreted as originated from the same, monoparental, giant gas cloud.

However, there exist both theoretical and observational results showing inconsistencies with this scenario: the age pattern of $\mathrm{OB}$ associations is irregular, with the youngest ones located well outside the expanding gas ring (Perrot \& Grenier 2003); the ki-

\footnotetext{
1 Instituto de Astrofísica de Andalucía, CSIC, Apartado 3004, E-18080, Granada, Spain; nestor@iaa.es, emilio@iaa.es, delgado@iaa.es.

2 Instituto de Astronomía, Universidad Nacional Autónoma de México, C. P. 04510, México D. F., México; felias@astroscu.unam.mx.

3 Facultad de Física, Departamento de Física Atómica, Molecular, y Nuclear, Universidad de Sevilla, Apartado 1065, 41080 Sevilla, Spain; jcc-famn@us.es.
}

nematics of the gas does not seem to be in agreement with the velocity field of the stellar component; and the modelization of gas dynamics is not compatible with the estimated radius of the stellar component, as well as with its range of ages (Perrot \& Grenier 2003; Moreno et al. 1999). Moreover, the analysis of the stellar spatial velocity diagram in the $U-V$ plane shows a clear bimodal distribution (Elias et al. 2006a). All these results make it difficult to imagine a single monoparental origin for the GB and suggest a GB formed by a spurious concatenation of different stellar subsystems whose formation processes were not necessarily connected among them. This point is particularly important for a full understanding of the mechanisms involved in the formation of the Milky Way. Can we design some kind of experiment able to answer this question? Here we propose to analyze the fractal structure of the stellar component of the GB in order to go deeper into its nature and origin. The base of this approach lies in the fact that the interstellar medium seems to show a fractal structure when observed at the scale of clouds (Bergin \& Tafalla 2007 and references therein) and a multiscaling behavior (Chappell \& Scalo 2001) when galactic scales are considered. Thus, stars forming from the same cloud should exhibit fractal patterns too if their birth places uniformly follow the densest regions (Elmegreen \& Elmegreen 2001). In this work we first develop a "reliable" algorithm to compute fractal dimensions of a sample of discrete points $(\S 2)$, and then we use it to study the distribution of stars in the GB $(\S 3)$. The main conclusions are summarized in $\S 4$.

\section{ESTIMATING THE FRACTAL DIMENSION}

Strictly speaking, a fractal is defined as an object whose Hausdorff dimension is larger than its topological dimension (Mandelbrot 1983). To estimate the Hausdorff dimension, authors use different working definitions that fit their methods and needs, and thus, there is not a unique definition of fractal dimension. When dealing with a distribution of points in space, it is very useful to use the so-called correlation dimension (Grassberger \& Procaccia 1983). This is a widely used method because of its robustness and because it is relatively easy to implement on real (experimental or observational) data. 


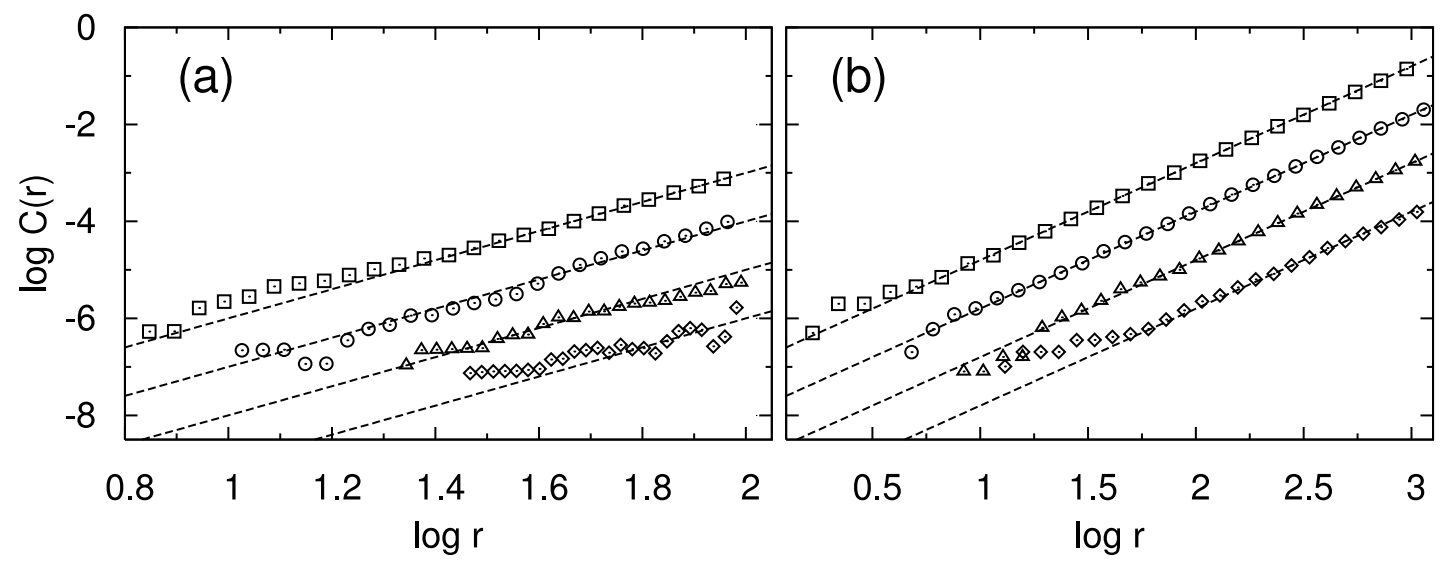

Fig. 1.-Correlation integral $C(r)$ for points distributed randomly within a disk of radius $R_{d}=2500 \mathrm{pc}$ and half-height $Z_{\max }=100$ pc. The number of points is $N=2000$ (squares), 1000 (circles), 500 (triangles), and 200 (rhombuses). All the lines have been arbitrarily shifted downward (except the top one) for clarity. (a) The calculation is done in the three-dimensional space using spheres with radius ranging from the minimal distance between two points to a maximum of $r_{\max }=Z_{\max }$. As a reference, the dashed lines show the expected slopes of 3 for these cases. For the case $N=200$ points, it is difficult to infer the expected linear behavior. $(b)$ Results when the calculation is done over the distributions projected on the $Z=0$ plane and using circles with different radii $r$ up to a maximum of $r_{\max }=R_{d} / 2$. In these cases, the expected value of the slopes (dashed lines) is 2 . The linear behaviors are clearly observed even for a relatively low number of points.

Let us consider a distribution of $N$ points in space with positions $x$. The number of other points within a sphere of radius $r$ centered on the $i$ th point is given by the expression

$$
n_{i}(r)=\sum_{j=1, j \neq i}^{N} H\left(r-\left|\boldsymbol{x}_{i}-\boldsymbol{x}_{j}\right|\right),
$$

where $H(x)$ is the Heaviside step function. This number can be evaluated by choosing $M$ different points as centers and then averaging to obtain the probability of finding a point within a sphere of radius $r$ centered on another point. This probability is expressed in the form

$$
C(r)=\frac{1}{M(N-1)} \sum_{i=1}^{M} n_{i}(r)
$$

For a fractal set, this quantity, called the correlation integral, scales at small $r$ as

$$
C(r) \sim r^{D_{c}},
$$

where $D_{c}$ is the correlation dimension, which in practice can be identified with the slope of the best fit in a $\log C(r)-\log r$ plot. In other words, the correlation dimension tells us how it changes (as $r$ increases) the probability that two points chosen randomly are separated by a distance smaller than $r$. For a homogeneous distribution of points in space we expect $D_{c}=3$, whereas in a plane $D_{c}=2$. If points are distributed obeying a fractal geometry, then $D_{c}<3$ (in three-dimensional space) or $D_{c}<2$ (in a plane).

Typically, when evaluating $D_{c}$ for real data (and not for infinite, perfect fractals) the power-law scaling relation (eq. [3]) is valid only within a limited range of $r$ values (Smith 1988). At very small scales (of the order of the mean distance to the nearest neighbor), the distribution looks like a set of isolated points, and $D_{c}$ tends to zero. On the other hand, the finite size of the set also results in decreased $D_{c}$ values at large $r$ values (of the order of the set size), because near the edge each point is surrounded by other points only on one side and then $C(r)$ tends to be underestimated. It has been proved that these and many other effects can lead to bad estimations of $D_{c}$, mainly when too few data are available (Smith 1988; Kitoh et al. 2000; Ciccotti \& Mulargia 2002).
In order to assess how the limited number of data points would alter our estimation of $D_{c}$ for the GB, we have done some numerical experiments. Figure 1 shows $\log (C)-\log (r)$ plots for random distributions of points within disklike structures. Boundary effects were avoided by keeping the condition that sampling spheres always are inside the volume occupied by the disk. For the three-dimensional case (Fig. 1a), the expected linear behavior is observed at high- $r$ values for a relatively high number of points $N$, but at low- $r$ values we see departures from the linear behavior arising from the lack of statistics of finite samples (Smith 1988). This tendency becomes more evident as $N$ decreases, because the mean distance between the nearest neighbors increases. For the case $N=200$ points, it is very difficult to infer a linear behavior at all. This is an important problem because many times the number of available data points is rather small. In these cases, the estimated fractal dimension depends strongly on the spatial range used to calculate the slope in the $\log (C)-\log (r)$ plot (Pisarenko \& Pisarenko 1995). However, when the calculation is done on the projected distributions (Fig. $1 b$ ), the range of $r$ values used to estimate the integral correlation can be extended to higher values, while still keeping the spheres (that actually become circles in the two-dimensional projection) inside the sample. In this case, the linear behavior is clearly appreciated - at relatively high $r$ values - even when only $N=200$ points are used. The average $D_{c}$ values for 10 random realizations, calculated by doing linear fits in the range $\log r \geq 1.5$, are shown in Table 1 . The results are always close to the theoretical values ( 3 or 2 ), but the standard deviations become quite high in the three-dimensional

TABLE 1

The Fractal Dimension for Random Point Distributions

\begin{tabular}{ccccc}
\hline \hline$N$ & $D_{c}(3 \mathrm{D})$ & $\sigma(3 \mathrm{D})$ & $D_{c}(2 \mathrm{D})$ & $\sigma(2 \mathrm{D})$ \\
\hline $2000 \ldots \ldots \ldots \ldots \ldots \ldots \ldots \ldots \ldots \ldots \ldots \ldots \ldots$ & 3.04 & 0.10 & 2.00 & 0.01 \\
$1000 \ldots \ldots \ldots \ldots \ldots \ldots \ldots \ldots \ldots \ldots \ldots \ldots$ & 3.05 & 0.27 & 2.00 & 0.03 \\
$500 \ldots \ldots \ldots \ldots \ldots \ldots \ldots \ldots \ldots \ldots$ & 3.13 & 0.59 & 1.98 & 0.04 \\
$200 \ldots \ldots \ldots \ldots \ldots \ldots \ldots$ & 3.12 & 1.32 & 2.08 & 0.14 \\
\hline
\end{tabular}

Notes.-The results are the average of 10 random realizations. Here we show (left to right columns) the number of points in each test distribution $(N)$ and the correlation dimension $\left(D_{c}\right)$ with its standard deviation $(\sigma)$ calculated both in the three-dimensional space (3D) and in the projected two-dimensional space (2D). 


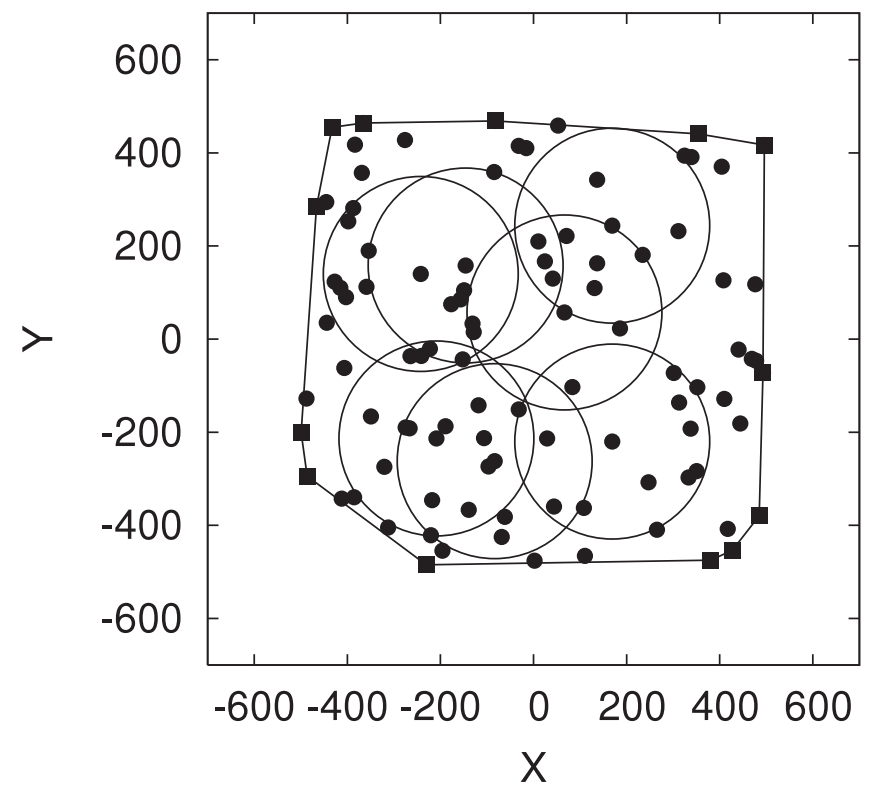

FIG. 2.-Example of random distribution of 100 points in a square region of side 1000 . The vertices of the convex hull (see text) are indicated by squares connected by lines. The circles (with radius 200) illustrate how the sampling is done by keeping them inside the boundary defined by the convex hull.

case for a relatively low number of points. In any case, the "problematic" region of relatively low $r$ values has to be excluded from the linear fit. In order to do this, we impose the condition that the standard deviation of each $C(r)$ value must be smaller than the corresponding $C(r)$ value. This simple criterion eliminates poorly estimated data (i.e., bad sampling) occurring mainly at $r$ values that are too low.

As an example, Figure 2 shows a random distribution of 100 points in a square region of side 1000 . In order to take into account edge effects, we first determine which points of the sample are vertices of the convex hull, i.e., we determine the minimumarea convex polygon containing the whole set of data points (square symbols connected by lines in Fig. 2). For this we use the algorithm proposed by Eddy (1977). Now we can place circles of different radii $r$ to evaluate $C(r)$ according to equation (2). The result is plotted in Figure 3. The calculations were carried out

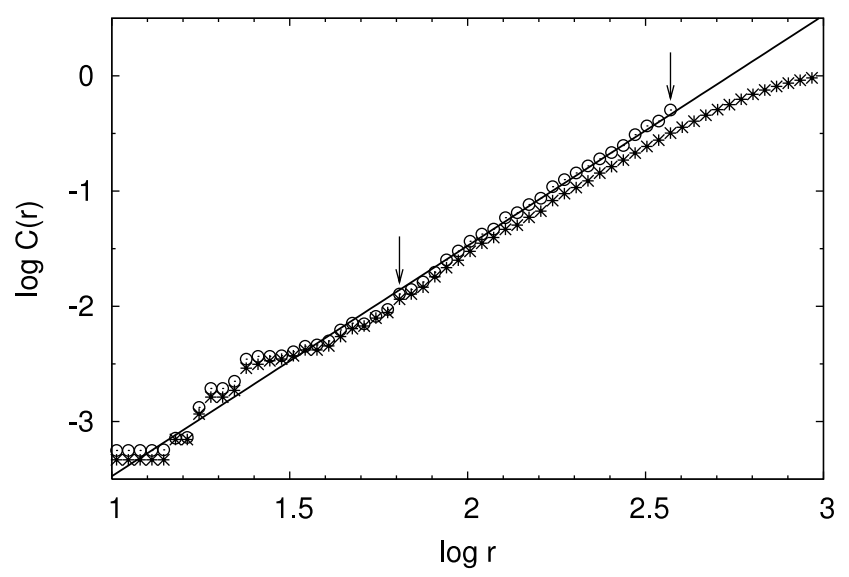

FIG. 3.-Correlation integral $C(r)$ for the same point set shown in Fig. 2, when the calculations are performed by taking (open circles) and not taking (asterisks) into account boundary effects. The solid line indicates the expected slope for a random distribution of points $\left(D_{c}=2\right)$, and the vertical arrows indicate the range for which the standard deviation of $C(r)$ is smaller than $C(r)$.

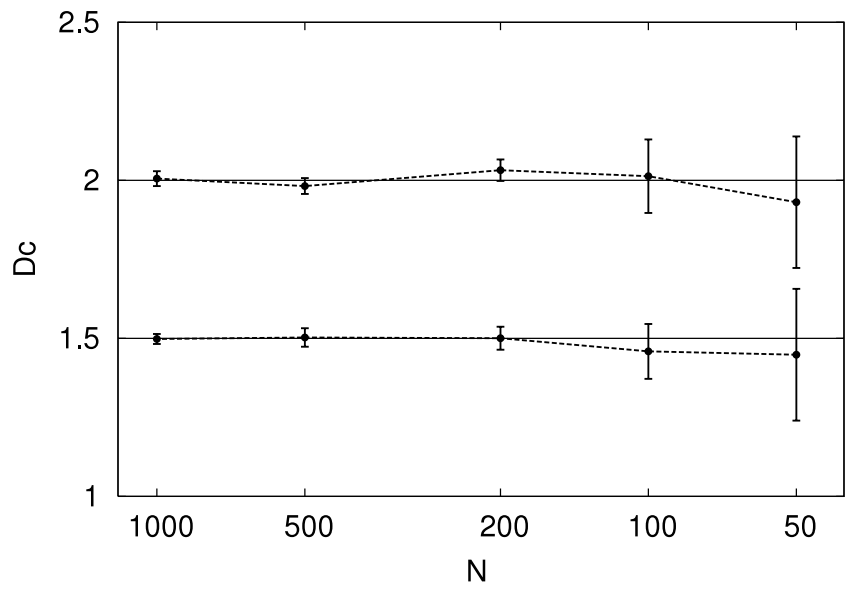

FIG. 4.-Correlation dimension $D_{c}$ as a function of the sample size $N$ for fractal distributions of points. Each result is the average of 10 different realizations, and the bars are the average of the uncertainties calculated using bootstrapping (see text). The horizontal lines indicate the fractal dimension used to generate the distribution of points (1.5 and 2).

both taking (open circles) and not taking (asterisks) into account boundary effects. If circles are allowed to cross boundaries, $C(r)$ tends to be underestimated, and this effect is higher the higher the radius $r$. For this reason, $D_{c}$ would tend to be smaller than the expected value if this effect is not considered (for comparison, the solid line in Fig. 3 shows the expected $D_{c}=2$ result). The range of $r$ values that fulfill the condition that the standard deviation of $C(r)$ is smaller than $C(r)$ is indicated in Figure 3 by vertical arrows. For $r$ values below this range, the departures from the linear behavior become more evident.

The algorithm we have developed follows the next steps. First, the three-dimensional distribution of points is projected on its mean plane; the boundary is determined by finding the convex hull of the sample; the correlation $C(r)$ is calculated using equations (1) and (2) but always keeping the circles inside the sample boundary; and then the correlation dimension is determined as the slope of the best $\log C(r)-\log r$ linear fit. Very low $r$ values [and consequently poorly estimated $C(r)$ values] are excluded from the fit. Finally, we use bootstrap techniques to estimate the uncertainty of the calculated value: we repeat the calculation of $D_{c}$ on a series of random resamplings of the data, and the standard deviation of the obtained set of fractal dimensions is taken as the error in our estimation. When working with relatively thin disks, the situation is practically the same as taking a two-dimensional slice of a spherical, three-dimensional distribution of points within a sphere. In this case, the fractal dimension of the threedimensional distribution of points $D_{c}(3 \mathrm{D})$ and the two-dimensional dimension $D_{c}(2 \mathrm{D})$ are related through the simple expression (Falconer 1990)

$$
D_{c}(2 \mathrm{D})=D_{c}(3 \mathrm{D})-1 \text {. }
$$

We have simulated two-dimensional fractal distributions of points in order to test the algorithm performance. The fractals were simulated by placing four squares of radius $R / L$ (with $L \geq 2$ ) inside a square of radius $R$ (we place one square in each quadrant). The procedure is repeated successively 10 times to obtain $4^{10}\left(\sim 10^{6}\right)$ points distributed according to a fractal pattern with dimension $D_{c}=$ $\log 4 / \log L$. Finally, we randomly removed points from the fractal until reaching a given sample size $N$. Figure 4 shows some example results for the cases $D_{c}=1.5(L \simeq 2.5)$ and $D_{c}=2(L=2)$. 


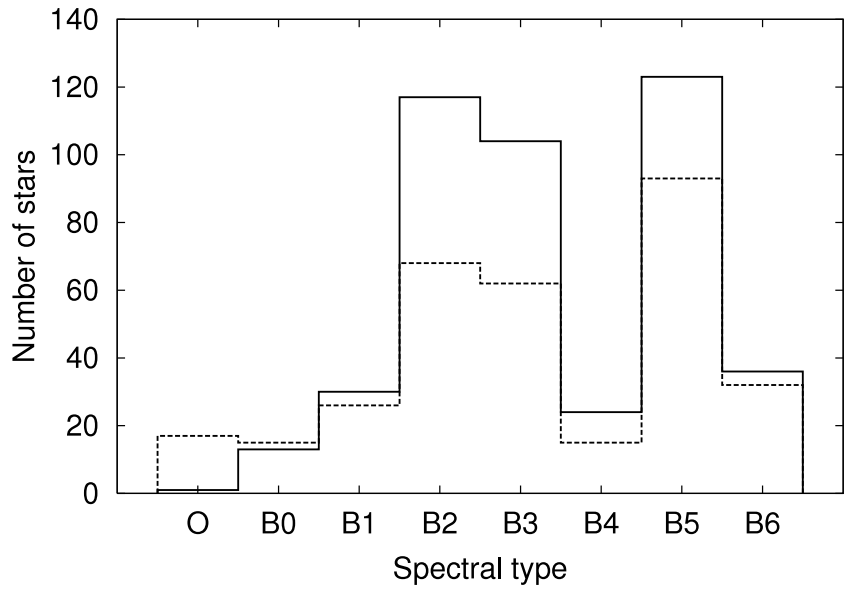

FIG. 5.- Distribution of spectral types for the sample of stars taken from Elias et al. (2006b). The solid-line histogram refers to stars belonging to the GB, whereas the dashed-line histogram refers to the LGD.

Even for relatively small sample sizes $(N \sim 100)$, the algorithm is able to estimate $D_{c}$ in a reliable way.

\section{THE FRACTAL DIMENSION OF THE GOULD BELT}

The first problem that arises when studying the GB is how to select the stars belonging to this system. Elias et al. (2006b) developed a new method to perform a three-dimensional spatial classification that was applied to a sample of $553 \mathrm{OB}$ stars from the Hipparcos catalog with precise distances of less than $1 \mathrm{kpc}$. This allowed them to separate and estimate the spatial structure of the stars belonging to the GB and to the LGD. The distributions of MK spectral types for the sample of stars used (Elias et al. 2006b) are shown in Figure 5. We see two populations clearly differentiated, one with spectral types earlier than B4 (which we call "early" stars) and another one with spectral types B4-B6 ("late" stars), that can be associated with two age groups centered at $\sim 20$ and $\sim 70 \mathrm{Myr}$, respectively. The spatial distribution of these stars is shown in Figure 6. The projections on each of the mean planes clearly reveal a clumpy, filamentary structure for the GB (Fig. $6 a$ ), whereas the stars in the LGD seem to be distributed more homogeneously (Fig. 6b). The gap almost free of stars seen in Figure 6 corresponds to the line of nodes in which both the GB and the LGD coexist. Close to this line, the probability of belonging to the GB has a high uncertainty, because the Bayesian probability in this region depends only on the "a priori" probability (i.e., the relative frequencies of both systems) and not on the probability conditioned to the spatial position (see Elias et al. 2006b). This gap is almost imperceptible for the GB and notorious for the LGD, simply because in the sample of stars used, there are more stars in the GB than in the LGD. What we have done in this work is to quantify the degree of inhomogeneity by calculating the fractal dimension ${ }^{4}$ for the GB and LGD (both early and late stars). The results are shown in Figure 7. For all cases, the behavior is almost perfectly linear in this log-log plot, with correlation coefficients $\simeq 0.99$. The number of circles used to evaluate $C(r)$ for each $r$ (i.e., $M$ in eq. [2]) is usually of the order of $10^{2}$. However, strictly speaking, $M$ depends on $r$ in the sense that larger $r$ allows fewer circles within the borders. For the largest $r$ values considered, $M$ is of the order of 10 . The slopes of the linear fits give the following fractal dimension values:

$$
\begin{cases}\text { GB-early, } & D_{c}(3 \mathrm{D})=2.68 \pm 0.04 \\ \text { GB-late }, & D_{c}(3 \mathrm{D})=2.85 \pm 0.04 \\ \text { LGD-early, } & D_{c}(3 \mathrm{D})=2.89 \pm 0.06 \\ \text { LGD-late, } & D_{c}(3 \mathrm{D})=2.84 \pm 0.06\end{cases}
$$

If the empty gap mentioned above was producing some bias in the determination of $D_{c}$ for the LGD, then the unbiased values should be even higher than the obtained ones.

We see that the distribution of stars in the solar neighborhood exhibits a certain degree of fractality, with $2.7 \lesssim D_{f} \lesssim 2.9$. Statistical tests show that there is a difference between early stars in the GB and the other subsets that is, in the worst of the cases,

${ }^{4}$ The term "fractal dimension" alone may be ambiguous, because there exist several different definitions of this quantity. We indifferently use the term "fractal dimension" or "correlation dimension" to refer to $D_{c}$.

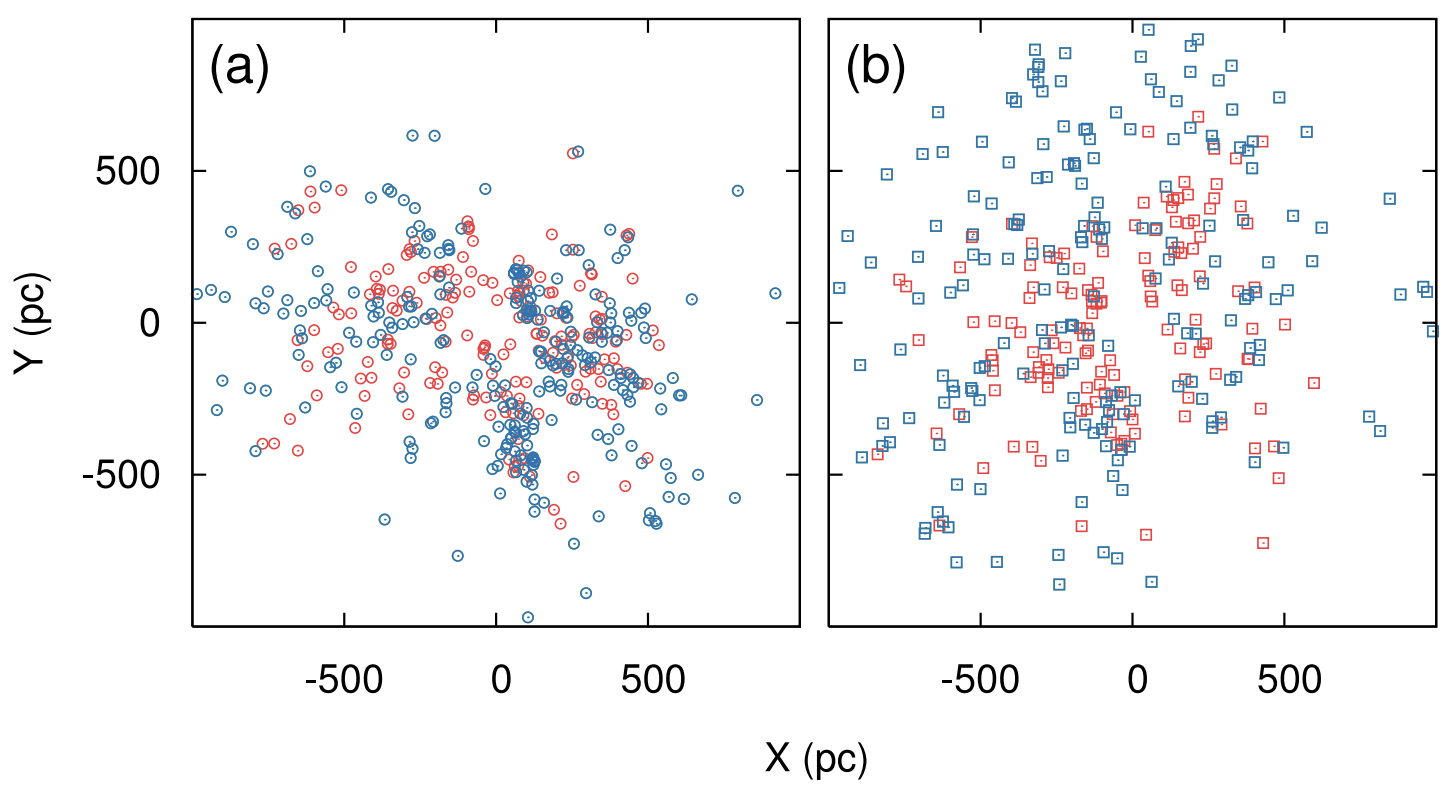

FIG. 6.- Positions of the stars in our sample. Stars are represented as circles (for the GB) or squares (for the LGD). The blue symbols refer to the spectral range O-B3, and red symbols refer to the range B4-B6. The panels show the projections on the mean planes for each distribution, both for the GB $(a)$ and for the LGD $(b)$. 


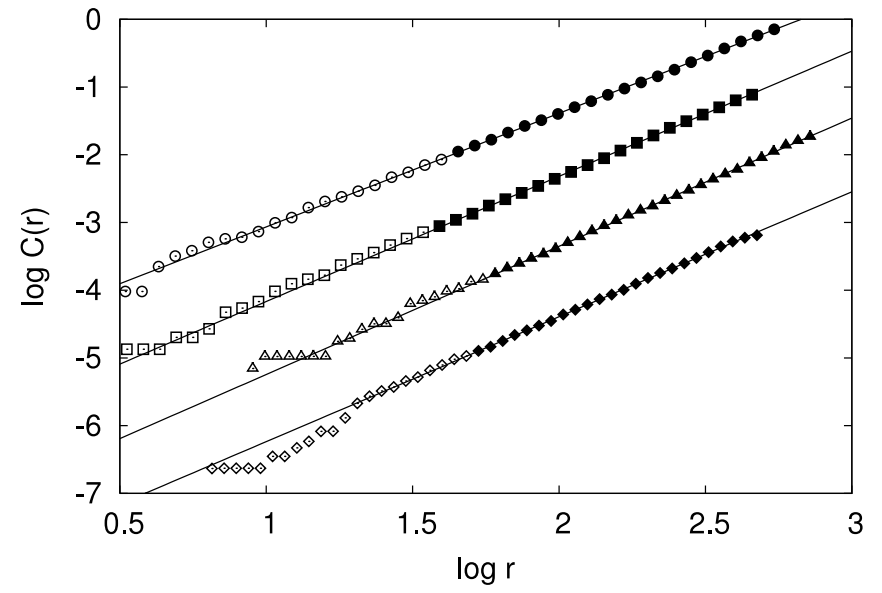

FIG. 7.-Projected correlation integral for stars in the solar neighborhood. The calculations were done for GB-early stars (circles), GB-late stars (squares), LGDearly stars (triangles), and LGD-late stars (rhombuses). Filled symbols indicate the range used for the linear fits (shown as lines). All the lines have been arbitrarily shifted downward (except the top one) for clarity.

statistically significant. Therefore, interestingly, the fractal dimension of early stars in the GB $\left(D_{f} \sim 2.7\right)$ is significantly smaller than that of the rest of the sample. Thus, these early stars have similar ages and are distributed following fractal patterns analogous to those observed in the gas of star-forming regions in the Galaxy (Sánchez et al. 2005, 2007). Therefore, it seems very likely that this group of stars was born from the same parent cloud. In contrast, later stars in the GB have a somewhat different, more homogeneous type of distribution with a fractal dimension similar to that obtained for the LGD $\left(D_{f} \gtrsim 2.85\right)$.

What is the origin of this difference? First, we have to point out that, in principle, different global properties are expected for the interstellar medium (ISM) at different spatial and/or temporal scales, because the main physical mechanisms acting at each scale are not necessarily the same. A monolithic gas cloud can be characterized as a turbulent medium, and this turbulence can be driven by many energy sources (Elmegreen \& Scalo 2004). However, at larger spatial scales, the ISM in a spiral arm is influenced by other disturbances (gravitational, magnetic, etc.) that modify the gas distribution. The fractal analysis gives us a simple but objective measurement of such structure through the degree of smoothness or clumpyness. This kind of analysis has been applied extensively to evaluate the structure of interstellar gas from parsec to kiloparsec scales, as well as the distribution of stars, star clusters, and star-forming sites (see Cartwright et al. 2006; Khalil et al. 2004; Westpfahl et al. 1999; Elmegreen et al. 2006; Cartwright \& Whitworth 2004; de la Fuente Marcos \& de la Fuente Marcos 2006a, 2006b, 2006c; Odekon 2006 for some recent examples). But often the analysis techniques are so diverse that it is not quite easy to extract robust conclusions. It seems that the average fractal dimension of single gas clouds is around $D_{f} \sim 2.7$ (Sánchez et al. 2005, 2007), although it may vary when the spatial scale is increased and when the distribution of gas clouds in the galactic disk is analyzed (Chappell \& Scalo 2001). But what about the stars? Young, newborn stars will reflect the conditions of the ISM from which they were formed. Therefore, a group of stars born from the same monolithic cloud, i.e., born at almost the same place and time, should have a fractal dimension similar to that of the parent cloud. This is exactly what we find for early-type stars in the GB: we obtain a fractal dimension very similar to the ISM value. Otherwise, if the star sample is representative of a population born from various clouds and/or with different star formation histories, then the fractal dimension will represent the gas distribution at different spatial or temporal scales according to a multiscale structure of the ISM (Chappell $\&$ Scalo 2001). At a galactic level, the fractal dimension of the distribution of stars and/or star-forming regions exhibits a very wide range of values, but until now no correlation has been clearly found between fractal patterns at the galactic level and other galactic properties (Odekon 2006; Feitzinger \& Galinski 1987; Parodi \& Binggeli 2003). There are, however, some suggestions that the fractal dimension of the distribution of stars and star-forming sites increases with time after the star formation process (de la Fuente Marcos \& de la Fuente Marcos 2006a, 2006b, 2006c; Odekon 2006; Schmeja \& Klessen 2006), probably due to the action of some physical mechanisms which tend to reorganize/destroy the original structure.

Here we have found quite different values for the fractal dimension of early-type stars in the GB and the LGD. This difference reflects the fact that most of the OB associations in the solar neighborhood are located in the GB (de Zeeuw et al. 1999; Elias et al. 2006b). That is, the distribution of OB stars in the LGD does not seem to show any kind of "typical" stellar grouping, whereas young OB stars in the GB exhibit a more hierarchized distribution with multiple OB associations. In this sense, we can say that the GB shows clear signatures of the internal structure of its parental cloud. This clustering in the young population of the GB is quantified through the fractal dimension, whose value is very close to the value characterizing gas clouds in the Galaxy. Thus, the possible causes for different values for the fractal dimension of early-type stars in the GB and the LGD can be studied by analyzing the possible causes for the different number of associations in both stellar systems. There are three possible factors (or a combination of them) that can contribute to this difference. First, the age difference between both groups could be significant enough to explain this result. It is clear that the separation according to the spectral type of the stars provides only a gross age separation. The spectral types allow us to derive an upper limit for the age, but they do not give the real lifetime of a star that also depends on the initial chemical composition. Although the earlier spectral groups of both the GB and the LGD have similar mean values and variances (see Fig. 5), there exists the possibility that one group is actually younger than the other one. This age difference could be enough to allow the LGD group to disperse showing a less structured distribution, whereas the GB group would still trace the density peaks of the parental cloud. Second, the difference in the degree of clustering among both groups can be produced by different physical conditions of the parental clouds. Much of stellar dispersal from active star-forming regions occurs on timescales of $10^{6}-10^{7} \mathrm{yr}$, and there are several possible scenarios to account for this dispersal of young stars from their birthplaces (Mamajek \& Feigelson 2001). The timescale for dispersal depends on the range of values of the density peaks inside the cloud which, at the same time, should depend on the local ISM pressure (Elmegreen 2006). Thus, clouds having different dynamical evolutions in different environmental conditions would likely give rise to stellar groups with different lifetimes. Depending on these critical values and on the age range of the stellar sample, we can get either a complex of associations or a uniform distribution of stars. Third, different gas distributions in the parental clouds can be responsible for variations in the fractal dimension among young stars in the GB and the LGD. For the $\mathrm{GB}, D_{f}$ agrees well with the range of values expected for the case of single monoparental clouds in the Galaxy, i.e., $D_{f} \simeq 2.5-2.7$ (Sánchez et al. 2005, 2007). On the other hand, the fact that the fractal dimension of the LGD is higher than the expected for 
individual clouds suggests that this population did not have a monoparental origin. Even though the young stars in the LGD were born in individual clouds, each one having internal fractal dimensions around $\sim 2.6$, the set of clouds that formed this group were distributed following a spatial pattern with a higher fractal dimension at a larger scale according to a multiscale scenario (Chappell \& Scalo 2001). In other words, even though the two young stellar groups in the GB and the LGD were born at the same time, the structure of the parental gas at the birth time has likely driven the final geometric structure of these stellar populations.

In order to restrict even more the problem under consideration, we have selected subsamples of stars with spectral types earlier than B2 both for the GB and for the LGD. Stars belonging to these groups have ages younger than $\sim 10 \mathrm{Myr}$. The determination of the fractal dimension for these "very early" subsamples yields similar results: $D_{c}(3 \mathrm{D})=2.62 \pm 0.08$ for the GB and $D_{c}(3 \mathrm{D})=2.84 \pm 0.08$ for the LGD. The uncertainties are higher than before because of the smaller number of stars in the samples. Obviously, this result does not rule out the possibility that both groups have different ages, but in this case, it is more difficult to interpret these results in terms of age differences among groups.

With the available data we cannot unambiguously discriminate the most likely explanation for the different fractal dimensions found for the very early-type stars in the GB and the LGD. Maybe all of these factors are contributing in some way to the problem. However, we consider that the main factors are probably related to different physical conditions in the parental clouds or different internal structure for the gas associated with a multiscale scenario. Finally, we want to mention that besides the different geometry and kinematics shown by the GB and the LGD (see Elias et al. 2006a, 2006b and references therein) the internal distribution of stars with spectral types earlier than B4 (quantified through the fractal dimension) allows us clearly to differentiate both systems. This difference could be explained in terms of a hierarchical star formation scenario.

\section{CONCLUSIONS}

We have developed an algorithm which uses equations (1) and (2) to estimate the correlation dimension of the stellar component of the GB and the LGD. The novelty of the algorithm lies in the implementation of objective criteria to avoid boundary effects and finite-data problems at small scales. We find that early OB stars (of spectral types earlier than B4) in the GB have a fractal dimension $\sim 2.7$. This values is very similar to that of the gas clouds in our Galaxy. This result seems to indicate that younger OB stars in the GB "preserve the memory" of the spatial structure of the cloud where they were born. On the contrary, stars in the GB of later spectral types show a larger fractal dimension, very similar to the value found for stars of both age groups in the LGD ( 2.8-2.9). Several factors (or a combination of them) can contribute to these morphological differences: age differences among the samples, different environmental conditions in the birth places, or different internal structure of the gas in the parental clouds.

We want to thank the referee for his/her valuable comments and criticisms that improved this paper. E. J. A. acknowledges funding from MEC of Spain through grants AYA 2004-05395 and AYA 2004-08260-C03-02 and from Consejería de Educación y Ciencia (Junta de Andalucía) through TIC-101.
Bergin, E. A., \& Tafalla, M. 2007, ARA\&A, in press (arXiv: 0705.3765) Cartwright, A., \& Whitworth, A. P. 2004, MNRAS, 348, 589

Cartwright, A., Whitworth, A. P., \& Nutter, D. 2006, MNRAS, 369, 1411

Chappell, D., \& Scalo, J. 2001, ApJ, 551, 712

Ciccotti, M., \& Mulargia, F. 2002, Phys. Rev. E, 65, 037201

de la Fuente Marcos, R., \& de la Fuente Marcos, C. 2006a, A\&A, 452, 163 2006b, A\&A, 454, 473

- 2006c, MNRAS, 372, 279

de Zeeuw, P. T., Hoogerwerf, R., de Bruijne, J. H. J., Brown, A. G. A., \& Blaauw, A. 1999, AJ, 117, 354

Eddy, W. F. 1977, Trans. Math. Soft., 3, 398

Efremov, Y. N. 1995, AJ, 110, 2757

Elias, F., Alfaro, E. J., \& Cabrera-Caño, J. 2006a, AJ, 132, 1052

Elias, F., Cabrera-Caño, J., \& Alfaro, E. J. 2006b, AJ, 131, 2700

Elmegreen, B. G. 2006, in ASP Conf. Ser., Mass Loss from Stars and the Evolution of Stellar Clusters, ed. A. de Koter, L. Smith, \& R. Waters (San Francisco: ASP), in press (astro-ph/0610679)

Elmegreen, B. G., Efremov, Y., Pudritz, R. E., \& Zinnecker, H. 2000, in Protostars and Planets IV, ed. V. Mannings, A. P. Boss, \& S. S. Russell (Tucson: Univ. Arizona Press), 179

Elmegreen, B. G., \& Elmegreen, D. M. 2001, AJ, 121, 1507

Elmegreen, B. G., Elmegreen, D. M., Chandar, R., Whitmore, B., \& Regan, M. 2006, ApJ, 644, 879

Elmegreen, B. G., \& Scalo, J. 2004, ARA\&A, 42, 211

Falconer, K. J. 1990, Fractal Geometry: Mathematical Foundations and Applications (London: Wiley)

Feitzinger, J. V., \& Galinski, T. 1987, A\&A, 179, 249

\section{REFERENCES}

Gould, B. A. 1879, Uranometria Argentina (Buenos Aires: Coni)

Grassberger, P., \& Procaccia, I. 1983, Phys. Rev. Lett., 50, 346

Guillout, P., Sterzik, M. F., Schmitt, J. H. M. M., Motch, C., \& Neuhauser, R. 1998, A\&A, 337, 113

Herschel, J. F. W. 1847, Results of Astronomical Observations Made during the

Years 1834-1838 at the Cape of Good Hope (London: Smith, Elder, \& Co.) Khalil, A., Joncas, G., \& Nekka, F. 2004, ApJ, 601, 352

Kitoh, S., Kimura, M., Mori, T., Takezawa, K., \& Endo, S. 2000, Phys. D, 141, 171

Lindblad, P. O., Grape, K., Sanqvist, A., \& Schober, J. 1973, A\&A, 24, 309

Mamajek, E. E., \& Feigelson, E. D. 2001, in ASP Conf. Ser. 244, Young Stars

Near Earth: Progress and Prospects, ed. R. Jayawardhana \& T. Greene (San Francisco: ASP), 104

Mandelbrot, B. B. 1983, The Fractal Geometry of Nature (New York: Freeman)

Moreno, E., Alfaro, E. J., \& Franco, J. 1999, ApJ, 522, 276

Odekon, M. C. 2006, AJ, 132, 1834

Parodi, B. R., \& Binggeli, B. 2003, A\&A, 398, 501

Perrot, C. A., \& Grenier, I. A. 2003, A\&A, 404, 519

Pisarenko, D. V., \& Pisarenko, V. F. 1995, Phys. Lett. A, 197, 31

Sánchez, N., Alfaro, E. J., \& Pérez, E. 2005, ApJ, 625, 849 2007, ApJ, 656, 222

Schmeja, S., \& Klessen, R. S. 2006, A\&A, 449, 151

Smith, L. A. 1988, Phys. Lett. A, 133, 283

Stothers, R., \& Frogel, J. A. 1974, AJ, 79, 456

Torra, J., Fernandez, D., \& Figueras, F. 2000, A\&A, 359, 82

Westpfahl, D. J., Coleman, P. H., Alexander, J., \& Tongue, T. 1999, AJ, 117, 868 